\title{
Real-world safety and effectiveness of nivolumab in Japanese patients with unresectable advanced or recurrent gastric/gastroesophageal junction cancer that has progressed after chemotherapy: a postmarketing surveillance study
}

\author{
Kensei Yamaguchi ${ }^{1}$ (D) Narikazu Boku ${ }^{2} \cdot$ Kei Muro $^{3} \cdot$ Kazuhiro Yoshida $^{4} \cdot$ Hideo Baba $^{5} \cdot$ Shinji Tanaka $^{6}$. \\ Ayumi Akamatsu ${ }^{6} \cdot$ Takeshi Sano $^{7}$
}

Received: 20 April 2021 / Accepted: 26 August 2021 / Published online: 28 September 2021

(c) The Author(s) 2021, corrected publication 2021

\begin{abstract}
Background This postmarketing surveillance study evaluated the real-world safety and effectiveness of nivolumab as salvage (after $\geq 2$ lines) therapy in Japanese patients with unresectable advanced or recurrent gastric/gastroesophageal junction (G/ GEJ) cancer.

Methods This multicenter, observational study was conducted at 158 centers in Japan. Patients with unresectable advanced or recurrent G/GEJ cancer were registered between Nov 1, 2017, and Oct 31, 2018, and observed for 6 months after treatment initiation with nivolumab. Correlation of background characteristics with treatment-related adverse events (TRAEs) and tumor response was explored.

Results Overall, 654 patients were registered (safety analysis set, $n=650$; effectiveness analysis set, $n=636$; response evaluation set, $n=516$ ). The incidences of all TRAEs and grade $\geq 3$ TRAEs were 31.5 and $11.2 \%$, respectively. TRAEs significantly correlated with the absence of peritoneal metastasis; C-reactive protein level < 1; prior G/GEJ cancer surgery; and past or concomitant pulmonary, thyroid, or renal disease (each $p<0.05$ ). The incidence of TRAEs was significantly lower in patients with higher Glasgow prognostic scores $(p<0.05)$. No new safety signals were observed. Complete response, partial response, stable disease, and progressive disease were observed in 1.2, 10.1, 27.1, and 58.3\% of the response evaluation set, respectively. Patients aged $\geq 65$ years $(13.9$ vs $5.3 \%, p=0.0083)$ and $\geq 75$ years $(18.8$ vs $9.2 \%, p=0.0036)$ showed a higher response rate than their younger counterparts.

Conclusions The real-world safety and effectiveness of nivolumab as salvage (after $\geq 2$ lines) therapy in Japanese patients with unresectable advanced or recurrent G/GEJ cancer were consistent with those observed in the phase 3 ATTRACTION-2 study.
\end{abstract}

Keywords Real world · Postmarketing surveillance $\cdot$ Gastric or gastroesophageal junction cancer $\cdot$ Nivolumab $\cdot$ Japan

Kensei Yamaguchi

kensei.yamaguchi@jfcr.or.jp

1 Department of Gastroenterological Chemotherapy, Cancer Institute Hospital of Japanese Foundation for Cancer Research, 3-8-31 Ariake, Koto-Ku, Tokyo 135-8550, Japan

2 Department of Gastrointestinal Medical Oncology, National Cancer Center Hospital, Tokyo, Japan

3 Department of Clinical Oncology, Aichi Cancer Center, Nagoya, Japan

4 Department of Surgical Oncology, Gifu University Graduate School of Medicine, Gifu, Japan
5 Department of Gastroenterological Surgery, Graduate School of Medical Sciences, Kumamoto University, Kumamoto, Japan

6 Pharmacovigilance Division, Ono Pharmaceutical Co., Ltd, Osaka, Japan

7 Department of Gastroenterological Surgery, Cancer Institute Hospital of Japanese Foundation for Cancer Research, Tokyo, Japan 


\section{Background}

Gastric cancer is the fifth most common cancer and the third leading cause of cancer-related deaths worldwide [1]. Gastric/gastroesophageal junction (G/GEJ) cancer accounts for $\sim 783,000$ deaths, with over 1,000,000 new cases of G/ GEJ cancer reported worldwide in 2018 [1]. The cumulative risk of developing gastric cancer from birth to age 74 years is higher in men (1.87\%) than in women $(0.79 \%)$ [1]. In Japan, gastric cancer was the third most common cause of cancer-related deaths in 2018 [2].

Nivolumab, an immune checkpoint inhibitor and a fully humanized anti-programmed cell death protein 1 (PD-1) monoclonal antibody, has been approved for various indications in more than 65 countries, including the United States, European Union, and countries in Asia [3]. In the ATTRAC TION-2 study, nivolumab, when compared with placebo in patients with unresectable advanced or recurrent G/GEJ cancer after failure of $\geq 2$ lines of chemotherapy [4], improved the overall survival (OS; median, 5.26 vs 4.14 months), with a better 1-year OS rate (26.2 vs $10.9 \%$ ) [4]. Consequently, the results of ATTRACTION-2 [4] led to the approval of nivolumab as a third- or later-line therapeutic option for patients with unresectable advanced or recurrent G/GEJ cancer in several countries, including Japan [5]. In Japan and Korea, nivolumab is recommended as third- or later-line therapy in the treatment guidelines of gastric cancer [5, 6].

However, as ATTRACTION-2 was limited by its sample size and stringent inclusion/exclusion criteria, the results from this study do not sufficiently reflect the real-world scenario, where not a few patients to be excluded from the clinical trials are treated with nivolumab. Accordingly, the Ministry of Health, Labour and Welfare (MHLW) required a postmarketing surveillance (PMS) study after drug approval. Therefore, this PMS study was conducted in Japan to evaluate the real-world safety and effectiveness of nivolumab in patients with unresectable advanced or recurrent G/GEJ cancer having diverse background factors. To the best of our knowledge, this is the first real-world evaluation of an immune checkpoint inhibitor in over 600 Japanese patients with unresectable advanced or recurrent G/GEJ cancer.

\section{Methods}

This PMS was a multicenter, open-label, observational study conducted at 158 centers in Japan. Patients with unresectable advanced or recurrent G/GEJ cancer to be treated with nivolumab $(n=654)$ were registered between Nov 1, 2017 , and Oct 31, 2018. These registered patients were observed for 6 months or until death after treatment initiation with nivolumab.

\section{Patients}

Patients with unresectable advanced or recurrent G/GEJ cancer who newly received nivolumab after failure of $\geq 2$ lines of chemotherapy were registered for this PMS study. Patients were excluded from the effectiveness analysis if they received nivolumab as off-label use (treatment for an indication other than unresectable advanced or recurrent $\mathrm{G} /$ GEJ cancer after failure of $\geq 2$ lines of chemotherapy before the initiation of nivolumab).

\section{Treatment}

Nivolumab at the approved dose as described in the prescribing information was administered. Of note, the approved dose was changed from the body weight-equivalent dose ( $3 \mathrm{mg} / \mathrm{kg}$ ) to a fixed dose of $240 \mathrm{mg} /$ body that received marketing authorization in August 2018. Treatment was discontinued based on the physician's discretion owing to progressive disease (PD) according to the Response Evaluation Criteria in Solid Tumors (RECIST) v1.1, lack of effectiveness even if the criteria for PD were not met, intolerability, or patients' refusal.

\section{Safety}

Adverse events (AEs) and treatment-related adverse events (TRAEs) were reported; their relation to nivolumab was judged by each attending physician. The clinical course of TRAEs of special interest was monitored. All AEs were graded using the National Cancer Institute Common Terminology Criteria for Adverse Events (NCI CTCAE v4.0 or v5.0).

\section{Effectiveness}

The question, "Was the response evaluated in accordance with the RECIST v1.1?," was included in the case report form (CRF). If the answer was "Yes," patients were included in the response evaluation set, in which tumor response [complete response (CR), partial response (PR), stable disease (SD), and PD] was assessed, and the objective response rate (ORR; CR or PR) was obtained. The 6-month survival rate was evaluated using the Kaplan-Meier method.

\section{Statistical analysis}

The sample size of the safety analysis set was planned at 500 , which provided a power of $77.74 \%$ to detect $\geq 1 \mathrm{AE}$ with a frequency of $0.30 \%$, corresponding to the lowest frequency of AEs that occurred in the ATTRACTION-2 trial $(0.30 \% ; 1 / 330$ patients). 
Exploratory subgroup analyses were performed on the incidence of TRAEs and the ORR; the comparison between subgroups was performed using Fisher's exact test, Wilcoxon rank sum test, or chi-squared test. Association between each patient characteristics, excluding the factors which allowed double count such as tumor location and histological differentiation, and the incidences of TRAEs and ORR were analyzed using multivariate logistic regression to adjust the number of nivolumab doses ( $\geq 5$ vs $\leq 4)$.

\section{Results}

\section{Patient disposition, demographics, and baseline characteristics}

A total of 654 patients were registered, of whom 650 comprised the safety analysis set and 636 comprised the effectiveness analysis set. Patient disposition is shown in Supplementary Fig. 1. Patient demographics and baseline characteristics of the safety analysis set are shown in Table 1. In the safety analysis set, $73 \%$ of patients were men; the median (range) age was 69 (20-87) years; $90 \%$ of patients had an Eastern Cooperative Oncology Group performance status (ECOG PS) of 0 or 1 ; and the median number of prior treatment regimens was $2(0-6)$. The number of patients with poorly differentiated adenocarcinoma and well-differentiated adenocarcinoma was 385 and 343, respectively, allowing a double count if a patient had histological components of both types. In addition, the proportion of patients with a large amount of ascites was $17.7 \%$ and those with past or present comorbidities was $65.8 \%$.

\section{Treatment}

A total of $460(70.8 \%)$ and $69(10.6 \%)$ patients received nivolumab at doses of 3 and $240 \mathrm{mg} /$ body intravenous infusion per cycle, respectively, throughout the study period. In $77(11.8 \%)$ patients, the dose was changed from 3 to $240 \mathrm{mg} /$ body during the study period at the physician's discretion, while 44 (6.8\%) patients received other doses. The mean \pm standard deviation (median; range) number of nivolumab administration was $5.7 \pm 4.1(4.5 ; 1-14)$ in the safety analysis set.

A total of $106(16 \%)$ patients continued treatment with nivolumab throughout the 6-month observation period. Among the remaining 544 (84\%) patients, the major reasons for nivolumab discontinuation within 6 months were disease progression (including death; 71.8\%), AEs (10.9\%), lack of effectiveness (8.9\%), and hospital transfer (1.1\%). One patient $(0.2 \%)$ discontinued treatment with nivolumab due to achieving a complete response as determined by the physician according to RECIST.
Table 1 Patient demographics and baseline characteristics (safety analysis set)

\begin{tabular}{|c|c|}
\hline & $\begin{array}{l}\text { Patients, } n(\%) \\
N=650\end{array}$ \\
\hline \multicolumn{2}{|l|}{ Age (1) (years) } \\
\hline 15 to $<65$ & $183(28.2)$ \\
\hline 65 to $<75$ & $301(46.3)$ \\
\hline$\geq 75$ & $166(25.5)$ \\
\hline \multicolumn{2}{|l|}{ Age (2) (years) } \\
\hline$\geq 65$ & 467 (71.9) \\
\hline \multicolumn{2}{|l|}{ Age (3) (years) } \\
\hline$<75$ & $484(74.5)$ \\
\hline \multicolumn{2}{|l|}{ Sex } \\
\hline Male & $474(72.9)$ \\
\hline Female & $176(27.1)$ \\
\hline \multicolumn{2}{|l|}{ ECOG PS } \\
\hline 0 & $265(40.8)$ \\
\hline 1 & $320(49.2)$ \\
\hline 2 & $55(8.5)$ \\
\hline 3 & $10(1.5)$ \\
\hline 4 & $0(0.0)$ \\
\hline \multicolumn{2}{|l|}{ ECOG PS } \\
\hline $0-1$ & $585(90.0)$ \\
\hline $2-4$ & $65(10.00)$ \\
\hline \multicolumn{2}{|l|}{ Smoking } \\
\hline Smoker & $338(52.0)$ \\
\hline Nonsmoker & $238(36.6)$ \\
\hline Unknown & $74(11.4)$ \\
\hline \multicolumn{2}{|c|}{ Any past or present comorbidity } \\
\hline No & $221(34.0)$ \\
\hline Yes & $428(65.8)$ \\
\hline Unknown & $1(0.2)$ \\
\hline \multicolumn{2}{|l|}{ Past or present renal disease } \\
\hline No & $610(93.9)$ \\
\hline Yes & $39(6.0)$ \\
\hline Unknown & $1(0.2)$ \\
\hline \multicolumn{2}{|c|}{ Past or present pulmonary disease } \\
\hline No & $591(90.9)$ \\
\hline Yes & $58(8.9)$ \\
\hline Unknown & $1(0.2)$ \\
\hline \multicolumn{2}{|l|}{ Past or present thyroid disease } \\
\hline No & $607(93.4)$ \\
\hline Yes & $41(6.3)$ \\
\hline Unknown & $2(0.3)$ \\
\hline \multicolumn{2}{|l|}{ Location $^{\mathrm{a}}$} \\
\hline Gastroesophageal junction & 94 \\
\hline Stomach & 543 \\
\hline Others & 8 \\
\hline Unknown & 7 \\
\hline \multicolumn{2}{|l|}{ Prior surgery } \\
\hline Absent & $266(40.9)$ \\
\hline Present & $384(59.1)$ \\
\hline
\end{tabular}


Table 1 (continued)

\begin{tabular}{ll}
\hline & Patients, $n(\%)$ \\
& $N=650$ \\
\hline Histological type ${ }^{\mathrm{a}, \mathrm{b}}$ & \\
Well-differentiated adenocarcinoma & 343 \\
Poorly differentiated adenocarcinoma & 385 \\
Others & 7 \\
Unknown & 4 \\
HER2 status & \\
Negative & $469(72.2)$ \\
Positive & \\
Unknown & $127(19.5)$ \\
Peritoneal metastasis & $54(8.3)$ \\
Absent & \\
Present & $299(46.0)$ \\
Amount of ascites & $351(54.0)$ \\
None or small amount & \\
Large amount & $528(81.2)$ \\
Unknown & $115(17.7)$ \\
NLR & $7(1.1)$ \\
$<2.5$ & \\
$\geq 2.5$ & $321(49.4)$ \\
Unknown & $320(49.2)$ \\
CRP (mg/dL) & $9(1.4)$ \\
Unknown & \\
Glasgow prognostic score & \\
0 & \\
1 & \\
Unknown & \\
\hline & \\
\hline &
\end{tabular}

$C R P$ C-reactive protein, ECOG PS Eastern Cooperative Oncology Group performance status, FISH fluorescence in situ hybridization, $H E R 2$ human epidermal growth factor receptor 2, IHC immunohistochemistry, NLR neutrophil to lymphocyte ratio

${ }^{a}$ The total number exceeds the number of the whole population because more than one category for tumor location and histological differentiation were checked in the case report form in some patients

${ }^{\mathrm{b}}$ Well-differentiated adenocarcinoma includes papillary adenocarcinoma and tubular adenocarcinoma, whereas poorly differentiated adenocarcinoma includes signet ring cell carcinoma and mucinous adenocarcinoma

${ }^{\mathrm{c}} \mathrm{IHC} 3+$ or $\mathrm{IHC} 2+$ and FISH +

\section{Safety}

TRAEs (all grades) were reported in 205 (31.5\%) patients (Fig. 1). The median (range) duration from initiating nivolumab to occurrence of TRAEs was $32(1-178)$ days. The most common TRAEs ( $>2 \%)$ were hypothyroidism $(4.2 \%)$, diarrhea (3.7\%), decreased appetite (2.9\%), rash $(2.5 \%)$, and malaise $(2.3 \%)$. Grade $\geq 3$ TRAEs were observed in $11.2 \%$ of patients, and the most common grade $\geq 3$ TRAEs were decreased appetite and diarrhea ( $0.9 \%$ each).

\section{TRAEs categorized by patient background characteristics}

Incidences of TRAEs categorized by patient background characteristics are shown in Supplementary Table 1. In the univariate analysis, the following subsets showed significantly higher incidences of TRAEs: those without vs with peritoneal metastasis (35.8 vs $27.9 \% ; p=0.0344$ ), C-reactive protein (CRP) level $<1.0$ vs $\geq 1.0$ (33.6 vs $25.6 \%$; $p=0.0455)$, prior surgical treatment for G/GEJ cancer (+) vs ( - ) (34.6 vs $27.1 \%$; $p=0.0482$ ), and neutrophil to lymphocyte ratio $<5 \mathrm{vs} \geq 5$ (33.4 vs $22.2 \% ; p=0.0281$ ). More remarkably, a significantly higher incidence of TRAEs was observed in patients with vs without past or present comorbidities [34.8\% (149/428) vs $25.3 \%$ $(56 / 221) ; p=0.0160]$, including pulmonary disease [48.3\% $(28 / 58)$ vs $29.9 \%(177 / 591) ; p=0.0071]$, thyroid disease [48.8\% (20/41) vs 30.3\% (184/607); $p=0.0225]$, and renal disease [48.7\% (19/39) vs 30.5\% (186/610); $p=0.0212]$, and patients with lower Glasgow prognostic scores $(p=0.0298)$. TRAEs related to the kidneys were observed in none and in $0.49 \%(3 / 610)$ of patients with and without past or present renal disease. TRAEs related to the lungs were observed in a higher proportion of patients with [8.62\% (5/58)] vs without [2.71\% (16/591)] past or present pulmonary disease. TRAEs related to the endocrine system were observed in a higher proportion of patients with [26.83\% (11/41)] vs without [4.45\% (27/607)] past or present thyroid disease (Supplementary Table 2). Detailed information of past and present renal, pulmonary, and thyroid comorbidities categorized using system organ class and preferred terms are summarized in Supplementary Tables 3, 4, and 5. Although the incidence of TRAEs was statistically significantly associated with number of treatment lines before initiating nivolumab $(p=0.0281)$ and number of doses $(p=0.0337)$, a clear linear relationship was not observed; the incidence showed a peak at 3 treatment lines and 9-12 doses.

The multivariate analysis for incidence of TRAEs according to background factors adjusted by the number of nivolumab doses $(\geq 5$ vs $\leq 4)$ showed a similar significant increase in the incidence of TRAEs among patients with vs without peritoneal metastasis $(p=0.0494)$; and patients with any past or present comorbidities $(p=0.0259)$, including pulmonary $(p=0.0055)$, thyroid $(p=0.0287)$, and renal $(p=0.0189)$ diseases (Table 2).

\section{Clinical course of patients with TRAEs of special interest}

The clinical course of patients with TRAEs of special interest is summarized in Table 3. Among different TRAEs, the 
Fig. 1 Incidence of treatmentrelated adverse events categorized by grade

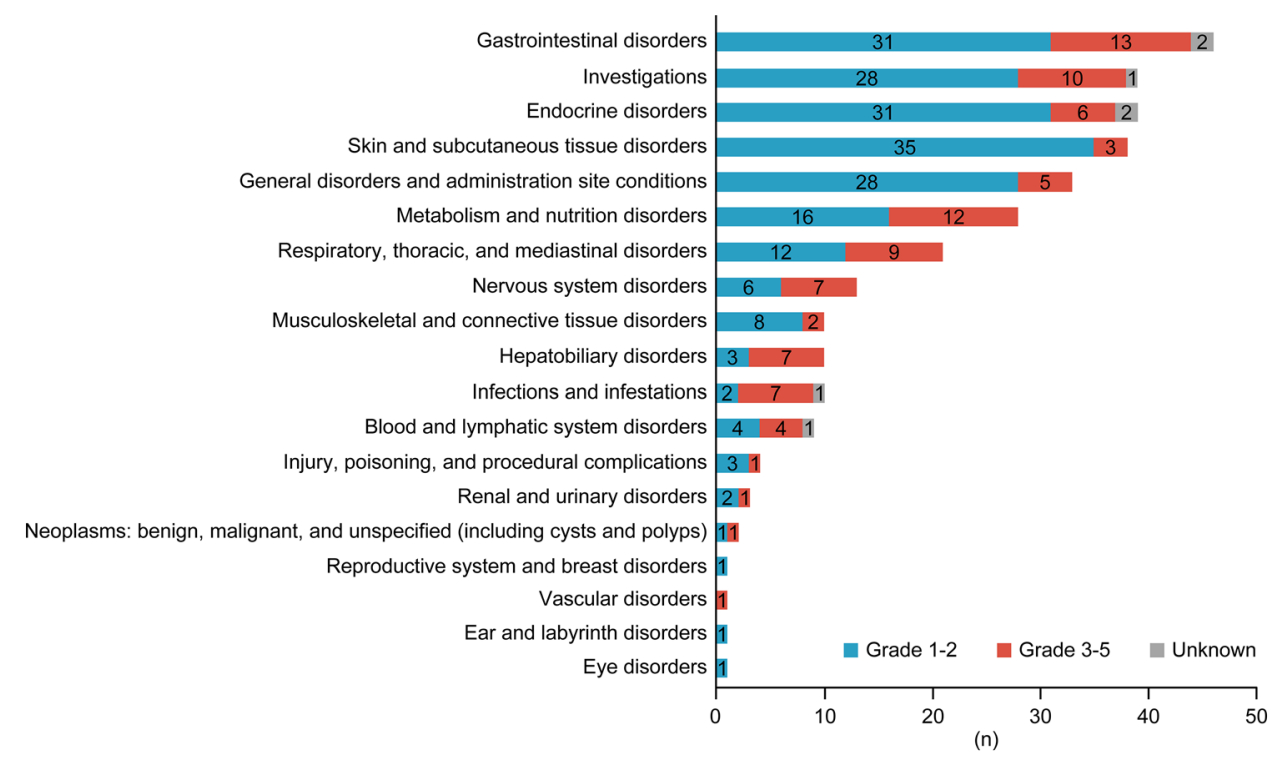

Table 2 Multivariate analysis for the risk of TRAEs according to background factors adjusted by the number of nivolumab doses ( $\geq 5$ vs $\leq 4$ times) (safety analysis set)

\begin{tabular}{|c|c|c|c|c|}
\hline Background factor $^{\mathrm{a}}$ & Comparison & Odds ratio & $95 \% \mathrm{CI}$ & $p$ value \\
\hline Age (1) & 65 to $<75$ years vs 15 to $<65$ years & 1.20 & $0.80-1.80$ & 0.3902 \\
\hline Age (2) & $\geq 75$ years vs 15 to $<65$ years & 1.44 & $0.91-2.28$ & 0.1172 \\
\hline Age (3) & $\geq 65$ vs $<65$ years & 1.28 & $0.87-1.87$ & 0.2050 \\
\hline Age (4) & $\geq 75$ vs $<75$ years & 1.29 & $0.88-1.87$ & 0.1872 \\
\hline ECOG PS & $2-4$ vs $0-1$ & 0.76 & $0.41-1.38$ & 0.3621 \\
\hline Smoking history & Smoker vs nonsmoker & 1.13 & $0.79-1.62$ & 0.5131 \\
\hline Any past or present comorbidity & Yes vs no & 1.52 & $1.05-2.19$ & $0.0259 *$ \\
\hline Past or present renal disease & Yes vs no & 2.19 & $1.14-4.20$ & $0.0189^{*}$ \\
\hline Past or present pulmonary disease & Yes vs no & 2.17 & $1.26-3.74$ & $0.0055^{*}$ \\
\hline Past or present thyroid disease & Yes vs no & 2.05 & $1.08-3.89$ & $0.0287^{*}$ \\
\hline Peritoneal metastasis & Yes vs no & 0.72 & $0.51-1.00$ & $0.0494 *$ \\
\hline Prior surgery for G/GEJ cancer & Yes vs no & 1.38 & $0.97-1.94$ & 0.0708 \\
\hline Location: GEJ & Yes vs no & 1.03 & $0.64-1.65$ & 0.9050 \\
\hline Location: stomach & Yes vs no & 0.98 & $0.63-1.53$ & 0.9216 \\
\hline Location: others & Yes vs no & 1.37 & $0.32-5.80$ & 0.6728 \\
\hline Histological type: well-differentiated adenocarcinoma ${ }^{\mathrm{b}}$ & Yes vs no & 1.28 & $0.92-1.79$ & 0.1494 \\
\hline Histological type: poorly differentiated adenocarcinoma ${ }^{c}$ & Yes vs no & 0.82 & $0.59-1.15$ & 0.2543 \\
\hline Histological type: others & Yes vs no & 1.46 & $0.32-6.64$ & 0.6221 \\
\hline Amount of ascites & Large vs none or small & 0.70 & $0.44-1.11$ & 0.1310 \\
\hline $\mathrm{CRP}^{\mathrm{d}}(\mathrm{mg} / \mathrm{dL})$ & $\geq 1.0 \mathrm{vs}<1.0$ & 0.74 & $0.50-1.10$ & 0.1364 \\
\hline Glasgow prognostic score ${ }^{\mathrm{d}}(1)$ & 1 vs 0 & 0.87 & $0.59-1.28$ & 0.4730 \\
\hline Glasgow prognostic score ${ }^{\mathrm{d}}(2)$ & 2 vs 0 & 0.65 & $0.40-1.06$ & 0.0815 \\
\hline $\mathrm{NLR}^{\mathrm{d}}$ & $\geq 2.5 \mathrm{vs}<2.5$ & 0.81 & $0.57-1.13$ & 0.2159 \\
\hline
\end{tabular}

$C I$ confidence interval, $C R P$ C-reactive protein, ECOG PS Eastern Cooperative Oncology Group performance status, GEJ gastroesophageal junction, NLR neutrophil to lymphocyte ratio, TRAE treatment-related adverse event

${ }^{a}$ Each multivariate analysis was assessed between nivolumab doses ( $\geq 5 \mathrm{vs} \leq 4$ times) and each background factor

${ }^{\mathrm{b}}$ Well-differentiated adenocarcinoma includes papillary adenocarcinoma and tubular adenocarcinoma

${ }^{c}$ Poorly differentiated adenocarcinoma includes poorly differentiated, signet ring cell carcinoma, and mucinous adenocarcinoma

${ }^{\mathrm{d}}$ Within 2 weeks before initiating nivolumab

${ }^{*} p<0.05$ was considered to be statistically significant 
recovery rate ranged from 0 to $82.1 \%$. Among patients who recovered, the median (range) time to recover was 100.0 (8-147) days in patients reporting thyroid dysfunction [19/35 (54\%)], 22.0 (2-121) days in patients reporting colitis or severe diarrhea [23/28 (82\%)], $29.0(14-120)$ days in patients reporting hepatic dysfunction [13/22 (59\%)], and 35.0 (17-170) days in patients reporting interstitial lung disease [11/15 (73\%)]. Deaths related to TRAEs of special interest occurred in 4 patients: colitis and severe diarrhea $(n=1)$, interstitial lung disease $(n=2)$, and venous thromboembolism $(n=1)$.

\section{Effectiveness}

Tumor response in the response evaluation set $(n=516)$ was CR in $6(1.2 \%)$ patients, PR in $52(10.1 \%)$ patients, SD in $140(27.1 \%)$ patients, PD in $301(58.3 \%)$ patients, and not evaluated (NE) in 17 patients (3.3\%). The 6-month survival rate was $52.0 \%$.

\section{ORR categorized by patient background characteristics}

ORR was assessed in subgroups categorized by patient background characteristics among 499 patients in the response evaluation set $(n=516)$ after excluding 17 patients assessed as "NE". Patients aged $\geq 65$ vs $<65$ years and $\geq 75$ vs $<75$ years showed a significantly higher ORR ( $p=0.0083$ and $p=0.0036$, respectively) (Supplementary Table 6). The multivariate analysis for tumor response according to background factors adjusted by the number of nivolumab doses ( $\geq 5$ vs $\leq 4)$ showed a significantly higher
ORR among patients in the higher age group $[\geq 75$ years vs 15 to $<65$ years $(p=0.0036) ; \geq 65$ vs $<65$ years $(p=0.0178) ; \geq 75$ vs $<75$ years $(p=0.0097)]$ (Table 4$)$. Objective response was achieved in 38 (18.5\%) patients who reported TRAEs [CR in $4(2.0 \%)$ patients and PR in 34 (16.6\%) patients] and in $30(6.7 \%)$ patients without TRAEs [CR in $3(0.7 \%)$ patients and PR in $27(6.1 \%)$ patients].

\section{Discussion}

This large PMS study was conducted to assess the real-world safety and effectiveness of nivolumab after failure of $\geq 2$ lines of chemotherapy in Japanese patients with unresectable advanced or recurrent G/GEJ cancer. The incidence of all TRAEs and grade $\geq 3$ TRAEs was 31.5 and $11.2 \%$, respectively. The occurrence of TRAEs categorized by system organ class was similar to that reported in the ATT RACTION-2 study [4], in which TRAEs of any grade were reported in $141(43 \%)$ patients in the nivolumab group, including 34 (10\%) with grade 3-4 TRAEs [4]. Clinical trials mandate a more detailed monitoring of adverse reactions compared with PMS studies, which potentially results in slightly higher incidences compared with those observed in clinical practice.

Patients with poor medical conditions, such as ascites and comorbidities, were excluded from the ATTRAC TION-2 study [4], and, therefore, the safety of nivolumab in these patients could not be evaluated. In this PMS, multivariate analysis showed that patients with past and present comorbidities such as pulmonary disease, thyroid disease, and renal disease, experienced significantly more TRAEs,

Table 3 Outcomes of TRAEs of special interest

\begin{tabular}{|c|c|c|c|c|c|c|c|}
\hline TRAEs of special interest & $N$ & $\begin{array}{l}\text { Resolved/resolving, } \\
n(\%)\end{array}$ & $\begin{array}{l}\text { Time to recovery, } \\
\text { (days) } \\
\text { Median (min-max) }\end{array}$ & $\begin{array}{l}\text { Recovered } \\
\text { with sequelae, } \\
n(\%)\end{array}$ & $\begin{array}{l}\text { Not recovered, } \\
n(\%)\end{array}$ & $\begin{array}{l}\text { Death, } \\
n(\%)\end{array}$ & $\begin{array}{l}\text { Unknown, } \\
n(\%)\end{array}$ \\
\hline Thyroid dysfunction & 35 & $19(54.3)$ & $100.0(8-147)$ & $0(0.0)$ & $13(37.1)$ & $0(0.0)$ & $3(8.6)$ \\
\hline Colitis and severe diarrhea & 28 & $23(82.1)$ & $22.0(2-121)$ & $0(0.0)$ & $4(14.3)$ & $1(3.6)$ & $0(0.0)$ \\
\hline Infusion reaction (within $24 \mathrm{~h}$ ) & 22 & $18(81.8)$ & $15.0(1-120)$ & $0(0.0)$ & $3(13.6)$ & $0(0.0)$ & $1(4.5)$ \\
\hline Hepatic function disorder & 22 & $13(59.1)$ & $29.0(14-120)$ & $0(0.0)$ & $8(36.4)$ & $0(0.0)$ & $1(4.6)$ \\
\hline Interstitial lung disease & 15 & $11(73.3)$ & $35.0(17-170)$ & $0(0.0)$ & $2(13.3)$ & $2(13.3)$ & $0(0.0)$ \\
\hline Renal disorder & 6 & $4(66.7)$ & $39.5(8-108)$ & $0(0.0)$ & $2(33.3)$ & $0(0.0)$ & $0(0.0)$ \\
\hline Adrenal disorder & 4 & $3(75.0)$ & $17.0(14-162)$ & $0(0.0)$ & $1(25.0)$ & $0(0.0)$ & $0(0.0)$ \\
\hline Venous thromboembolism & 2 & $1(50.0)$ & $22.0(22-22)$ & $0(0.0)$ & $0(0.0)$ & $1(50.0)$ & $0(0.0)$ \\
\hline Type 1 diabetes mellitus & 2 & $0(0.0)$ & - & $1(50.0)$ & $1(50.0)$ & $0(0.0)$ & $0(0.0)$ \\
\hline Sclerosing cholangitis & 1 & $0(0.0)$ & - & $0(0.0)$ & $1(100.0)$ & $0(0.0)$ & $0(0.0)$ \\
\hline Nerve disorder & 1 & $0(0.0)$ & - & $0(0.0)$ & $1(100.0)$ & $0(0.0)$ & $0(0.0)$ \\
\hline $\begin{array}{l}\text { Myasthenia gravis, myocarditis, } \\
\text { myositis, and rhabdomyolysis }\end{array}$ & 1 & $0(0.0)$ & - & $1(100.0)$ & $0(0.0)$ & $0(0.0)$ & $0(0.0)$ \\
\hline
\end{tabular}

max maximum, min minimum, TRAE treatment-related adverse event 
Table 4 Multivariate analysis for tumor response according to background factors adjusted by the number of nivolumab doses ( $\geq 5$ vs $\leq 4$ times) (response evaluation set: excluding patients whose tumor response was NE; patients whose evaluation method complied with RECIST v1.1)

\begin{tabular}{lllll}
\hline Background factor $^{\text {a }}$ & Comparison & Odds ratio & $95 \%$ CI & $p$ value \\
\hline Age (1) & 65 to $<75$ years vs 15 to $<65$ years & 2.19 & $0.91-5.26$ & 0.0810 \\
Age (2) & $\geq 75$ years vs 15 to $<65$ years & 3.83 & $1.55-9.47$ & $0.0036^{*}$ \\
Age (3) & $\geq 65$ vs $<65$ years & 2.74 & $1.19-6.31$ & $0.0178^{*}$ \\
Age (4) & $\geq 75$ vs $<75$ years & 2.18 & $1.21-3.94$ & $0.0097^{*}$ \\
ECOG PS & 2-4 vs 0-1 & 1.24 & $0.34-4.53$ & 0.7448 \\
Smoking history (past) & Smoker vs nonsmoker & 1.86 & $0.99-3.47$ & 0.0525 \\
Amount of ascites & Large vs none or small & 1.34 & $0.58-3.10$ & 0.4951 \\
NLR & & 1.20 & $0.68-2.13$ & 0.5355 \\
\hline
\end{tabular}

CI confidence interval, ECOG PS Eastern Cooperative Oncology Group performance status, NLR neutrophil to lymphocyte ratio, RECIST Response evaluation criteria in solid tumors

${ }^{a}$ Each multivariate analysis was conducted between nivolumab doses ( $\geq 5$ vs $\leq 4$ times) and each background factor

${ }^{\mathrm{b}}$ Within 2 weeks before initiating nivolumab

${ }^{*} p<0.05$ was considered to be statistically significant suggesting that comorbidities likely affect the overall incidence of TRAEs in patients treated with nivolumab. Although it is difficult to explain how past and present renal comorbidities affect renal TRAEs, TRAEs in the lungs and endocrine system were observed in a higher proportion of patients with vs without past or present pulmonary disease and thyroid disease, respectively, suggesting that pulmonary and thyroid comorbidities might be risk factors for pulmonary and endocrine TRAEs, respectively. These data from patients with comorbidities in this PMS may be useful to call attention to TRAEs of nivolumab in clinical practice.

On the contrary, patients with a good condition, such as no peritoneal metastasis, Glasgow prognostic score of 0 , CRP level $<1$, and prior surgical treatment for G/GEJ cancer, are likely to experience significantly more TRAEs. Moreover, higher incidences of TRAEs in patients with no peritoneal metastasis and a Glasgow prognostic score of 0 and CRP level $<1$ were associated with an increased exposure period of nivolumab. The multivariate analysis adjusted by the treatment period identified the absence of peritoneal metastasis and any past or present comorbidities as factors associated with TRAEs. Both the absence of peritoneal metastasis and a Glasgow prognostic score of 0 are wellknown favorable prognostic factors. It is considered that the active immune status in patients with a good condition may be related to TRAEs. About 54-82\% of patients who experienced TRAEs of special interest were resolved/resolving by pharmacotherapy such as corticosteroids and hormone replacement or by observation only. As some TRAEs were not resolved, leading to sequelae and/or death, early diagnosis and optimal treatment for TRAEs are very important in clinical practice.

Tumor response among the response evaluation set (CR, $1.2 \%$; PR, 10.1\%; SD, 27.1\%; and PD, 58.3\%) and the 6 -month survival rate $(52.0 \%)$ were consistent with those reported in the ATTRACTION-2 trial [4]. Reportedly, the occurrence of TRAEs was associated with better efficacy of nivolumab in patients with metastatic melanoma [7] and advanced G/GEJ cancer [8]. In the CheckMate 141 study in patients with recurrent or metastatic head and neck cancer receiving nivolumab, the median OS (14.3 vs 5.2 months; hazard ratio, $0.10 ; 95 \%$ confidence interval, $0.03-0.39)$ and 2-year OS rates (31.3 vs $0 \%$ ) were higher in patients with TRAEs, particularly skin-related disorders, than in those without TRAEs [9]. Similarly, the ORR (18.5 vs 6.7\%) was higher in patients who reported TRAEs than in those without TRAEs in this PMS study. When ORR was compared according to patient background characteristics in this PMS, ORR increased with increasing age from $\geq 50$ years. However, in the ATTRACTION-2 trial, there was no clear relationship between age and response. Thus, the reason for a higher ORR in the elderly remains unknown. Biological and/or immunological background, such as tumor mutation burden, might be confounding in this PMS study. Furthermore, the limited number of patients in the categories by age may have impacted data interpretation. It is expected that biomarkers for efficacy and TRAEs will be established in the future.

As is the nature of PMS studies, clinical information could not be collected as precisely as in the clinical trials, and the observation period was rather short. Additionally, no biomarker analysis was conducted. A comparison between subgroups could be affected by factors that were not collected or evaluated. Also, multivariate analysis could not be performed for factors such as tumor location and histological differentiation because some patients were counted repeatedly for each tumor location and differentiation. However, this study could cover a wide population of patients with unresectable advanced or recurrent G/ GEJ cancer receiving nivolumab, and the obtained results 
can provide useful information reflecting the real-world scenario.

\section{Conclusions}

The results of this PMS study showed that the safety and effectiveness of nivolumab as salvage (after $\geq 2$ lines) therapy in Japanese patients with unresectable advanced or recurrent G/GEJ cancer was consistent with that observed in the phase 3 ATTRACTION-2 study. No new safety concerns were reported over the 6-month observation period.

Supplementary Information The online version contains supplementary material available at https://doi.org/10.1007/s10120-021-01244-y.

Acknowledgements Editorial support, in the form of medical writing, assembling tables and creating high-resolution images based on the authors' detailed directions, collating author comments, copyediting, fact-checking, and referencing, was provided by Annirudha Chillar, $\mathrm{MD}, \mathrm{PhD}$, of Cactus Life Sciences (part of Cactus Communications), and funded by Ono Pharmaceutical Co., Ltd. and Bristol-Myers Squibb.

Author contributions All authors except for Shinji Tanaka contributed to the study conception and design. Data collection and analysis were performed by Ayumi Akamatsu. Interpretation of the results was performed by Kensei Yamaguchi, Narikazu Boku, Ayumi Akamatsu, and Shinji Tanaka. All authors commented on previous versions of the manuscript, read and approved the final manuscript.

\section{Declarations}

Conflict of interest KYa has received research funding from Ono Pharmaceutical Co., Ltd., and Bristol-Myers Squibb for the work under consideration for publication. KYa has also received grants from Taiho Pharmaceutical, Yakult Honsha, Sanofi, Ono Pharmaceutical Co., Ltd., and Elli Lilly; and honoraria from Taiho Pharmaceutical, Chugai Pharmaceutical, Ono Pharmaceutical Co., Ltd., Eli Lilly, Bristol-Myers Squibb Japan, Merck Serono, and Takeda Pharmaceutical outside the submitted work. NB has received research funding from Ono Pharmaceutical Co., Ltd., and Bristol-Myers Squibb for the work under consideration for publication. NB has also received grants from Takeda Pharmaceutical and Ono Pharmaceutical Co., Ltd.; and honoraria for lectures from Ono Pharmaceutical Co., Ltd., Taiho Pharmaceutical, and Bristol-Myers Squibb outside the submitted work. KM has received grants from Ono Pharmaceutical Co., Ltd. and BristolMyers Squibb for the work under consideration for publication. KM has also received grants and personal fees from Amgen (research funding and consulting fee), Ono Pharmaceutical Co., Ltd. (research funding, consulting fee, and honorarium), Sanofi (research funding and honorarium), and Taiho Pharmaceutical (research funding and honorarium); grants (research funding) from Solasia Pharma, Merck Serono, Daiichi Sankyo, Parexel International, Pfizer, and MSD; and personal fees from AstraZeneca (consulting fee), Chugai Pharmaceutical (honorarium), Takeda Pharmaceutical, Eli Lilly, Bristol-Myers Squibb, and Bayer outside the submitted work. KYo has received grants and personal fees from Ono Pharmaceutical Co., Ltd. and Bristol-Myers Squibb for the work under consideration for publication. KY has also received grants and personal fees from Asahi Kasei Pharma, Chugai Pharmaceutical, Covidien Japan, Daiichi Sankyo, Eli Lilly Japan, Johnson and Johnson, Merck Serono, MSD, Nippon Kay- aku, Novartis Pharma, Sanofi, Taiho Pharmaceutical, Takeda Pharmaceutical, Tsumura and Co., and Yakult Honsha; grants from Abbott, AbbVie, Astellas, Biogen Japan, Celgene, Eisai, EP-CRSU, Fujifilm, GlaxoSmithKline K.K., Kaken Pharmaceutical, Kyowa Kirin, Meiji Seika Pharma, Otsuka Pharmaceutical, Koninklijke Philips, and Toray Medical; and personal fees from AstraZeneca, Denka Co., Ltd., EA Pharma, Olympus, Pfizer, Sanwa Kagaku Kenkyusho, SBI Pharma, Teijin Pharma, and Terumo outside the submitted work. HB has received grants from Ono Pharmaceutical Co., Ltd. and Bristol-Myers Squibb for the work under consideration for publication. HB has also received grants and personal fees from Ono Pharmaceutical Co., Ltd. and Taiho Pharmaceutical; grants from Merck Biopharma Co., Ltd. and MSD K.K.; and personal fees from Eli Lilly Japan K.K. outside the submitted work. AA and ST are employees of and own stocks in Ono Pharmaceutical Co., Ltd. TS has received research funding from Ono Pharmaceutical Co. Ltd., and Bristol-Myers Squibb for the work under consideration for publication. TS has also received lecture fees from Ono Pharmaceutical Co. Ltd., Taiho Pharmaceutical, Eli Lilly, Chugai Pharmaceutical, EA Pharma, Daiichi Sankyo, Takeda Pharmaceutical, and Nippon Kayaku outside the submitted work.

Ethical approval All procedures followed were in accordance with the ethical standards of the responsible committee on human experimentation (institutional and national) and with the Helsinki Declaration of 1964 and later versions. This was a noninterventional study conducted in routine clinical practice; therefore, in accordance with good postmarketing study practice regulations, written informed consent of patients was not required.

Open Access This article is licensed under a Creative Commons Attribution 4.0 International License, which permits use, sharing, adaptation, distribution and reproduction in any medium or format, as long as you give appropriate credit to the original author(s) and the source, provide a link to the Creative Commons licence, and indicate if changes were made. The images or other third party material in this article are included in the article's Creative Commons licence, unless indicated otherwise in a credit line to the material. If material is not included in the article's Creative Commons licence and your intended use is not permitted by statutory regulation or exceeds the permitted use, you will need to obtain permission directly from the copyright holder. To view a copy of this licence, visit http://creativecommons.org/licenses/by/4.0/.

\section{References}

1. Bray F, et al. Global cancer statistics 2018: GLOBOCAN estimates of incidence and mortality worldwide for 36 cancers in 185 countries. CA Cancer J Clin. 2018;68:394-424.

2. Latest cancer statistics. https://ganjoho.jp/reg_stat/statistics/stat/ summary.html

3. Bristol-Myers Squibb withdraws European application of Opdivo (nivolumab) plus Yervoy (ipilimumab) for the first-line treatment of advanced non-small cell lung cancer. https://news.bms.com/ press-release/corporatefinancial-news/bristol-myers-squibb-withd raws-european-application-opdivo-niv and https://www.ono.co.jp/ eng/news/pdf/sm_cn200124.pdf

4. Kang YK, Boku N, Satoh T, Ryu MH, Chao Y, Kato K, et al. Nivolumab in patients with advanced gastric or gastro-oesophageal junction cancer refractory to, or intolerant of, at least two previous chemotherapy regimens (ONO-4538-12, ATTRAC TION-2): a randomised, double-blind, placebo-controlled, phase 3 trial. Lancet. 2017;390:2461-71. 
5. Ono Pharmaceutical Co., Ltd. Press Release. September 22, 2017. https://www.ono-pharma.com/sites/default/files/en/news/press/ sm_cn170922.pdf. Accessed Jan 1, 2021.

6. Guideline Committee of the Korean Gastric Cancer Association (KGCA), Development Working Group and Review Panel. Korean practice guideline for gastric cancer. an evidence-based, multidisciplinary approach. J Gastric Cancer. 2018;2019(19):1-48.

7. Freeman-Keller M, Kim Y, Cronin H, Richards A, Gibney G, Weber JS, et al. Nivolumab in resected and unresectable metastatic melanoma: characteristics of immune-related adverse events and association with outcomes. Clin Cancer Res. 2016;22:886-94.

8. Masuda K, Shoji H, Nagashima K, Yamamoto S, Ishikawa M, Imazeki $\mathrm{H}$, et al. Correlation between immune-related adverse events and prognosis in patients with gastric cancer treated with nivolumab. BMC Cancer. 2019;19:974.

9. Yen CJ, Kiyota N, Hanai N, Takahashi S, Yokota T, Iwae S, et al. Two-year follow-up of a randomized phase III clinical trial of nivolumab vs the investigator's choice of therapy in the Asian population for recurrent or metastatic squamous cell carcinoma of the head and neck (CheckMate 141). Head Neck. 2020;42:2852-62.

Publisher's Note Springer Nature remains neutral with regard to jurisdictional claims in published maps and institutional affiliations. 